\title{
Endoscopic Ultrasound (EUS)-Guided Pancreatic Duct Drainage: The Basics of When and How to Perform EUS-Guided Pancreatic Duct Interventions
}

\author{
Christopher G. Chapman, Irving Waxman and Uzma D. Siddiqui \\ Center for Endoscopic Research and Therapeutics (CERT), University of Chicago Medicine, Chicago, IL, USA
}

Despite the advances in endoscopy, endoscopic ultrasound-guided pancreatic duct drainage (EUS-PDD) remains a technically challenging procedure. Technical success rates are greater than $70 \%$; however, the average rate of adverse events is nearly $20 \%$, which increases to $55 \%$ when stent migration is included. Until recently, a significant difficulty with this technique was the absence of dedicated devices. Proper patient selection is of utmost importance, and EUS-PDD should be reserved for patients who have failed endoscopic retrograde pancreatography. Furthermore, EUS-PDD must be performed by experienced endoscopists who are familiar with the technique. The most common indications include chronic pancreatitis induced strictures and stones, disconnected pancreatic ducts, inaccessible ampulla, and post-surgical altered anatomy. This manuscript will review the accessories used, techniques employed, and published literature reporting outcomes as well as adverse events regarding EUS-PDD. Clin Endosc 2016;49:161-167

Key Words: Endosonography; Stents; Pancreatic ducts; Drainage; Pancreatic duct intervention

\section{INTRODUCTION}

Endoscopic ultrasound-guided pancreatic duct drainage (EUS-PDD) has emerged as a therapeutic option to drain the pancreatic ducts (PDs) when conventional transpapillary endoscopic retrograde pancreatography (ERP) is unsuccessful. Although EUS-PDD may eliminate the need for more invasive procedures and surgery, the procedure itself remains technically challenging. It can be successfully and safely performed in the hands of adequately trained and experienced endoscopists, who limit its use to appropriately selected patients. The most common indications for EUS-PDD include

Received: December 12, 2015 Revised: January 11, 2016

Accepted: January 14, 2016

Correspondence: Uzma D. Siddiqui

Center for Endoscopic Research and Therapeutics (CERT), The University of Chicago Medicine and Biological Sciences, 5700 S Maryland Ave. MC 8043, Chicago, IL 60637, USA

Tel: +1-773-702-5997, Fax: +1-773-834-8891, E-mail: usiddiqui@bsd.uchicago.edu

(c) This is an Open Access article distributed under the terms of the Creative Commons Attribution Non-Commercial License (http://creativecommons.org/ licenses/by-nc/3.0) which permits unrestricted non-commercial use, distribution, and reproduction in any medium, provided the original work is properly cited. chronic pancreatitis induced strictures and stones, disconnected PDs, inaccessible ampullas, and postsurgical altered anatomy. This manuscript will review the indications, accessories used, techniques employed, and published literature reporting outcomes as well as adverse events regarding EUS-PDD.

\section{INDICATIONS}

\section{Chronic pancreatitis}

A frequent feature of chronic pancreatitis is unremitting abdominal pain that is multifactorial in etiology, but is believed to develop in part due to pancreatic ductal hypertension resulting from outflow obstruction. The pancreatic ductal hypertension is a sequela of parenchymal destruction, inflammation, and pathological fibrosis resulting in benign strictures of the main PD, papillary stricture, PD stones, rupture of the main PD, or a combination of these. Endoscopic therapy in chronic pancreatitis can provide benefit by decompressing the PD with stricture dilation, stent placement or stone fragmentation or removal. 


\section{Strictures}

The management of PD strictures requires a multi-disciplinary approach considering medical, endoscopic, and surgical therapeutic options. Although randomized controlled trials have demonstrated that surgery affords better long term pain control than endoscopic approaches, endoscopic therapy does not preclude future surgery, is less invasive, and is associated with lower rates of morbidity and mortality. ${ }^{1}$ Endoscopic therapy for strictures includes stricture dilation and stent placement. The technical success rate of pancreatic stent placement in patients with chronic pancreatitis has been reported to range from $85 \%$ to $98 \%$. Tight strictures that are non-amenable to typical endotherapeutic decompression via ERP may require EUS-PDD. In one of the largest case series, 36 patients underwent EUS-guided pancreatic drainage, and the most frequent indication was chronic pancreatitis (20 patients) with tight stricture (25\%, 9/36 cases). ${ }^{2}$

\section{Stones}

PD calculi are observed in up to $90 \%$ of patients with chronic pancreatitis and may cause PD obstruction contributing to abdominal pain or recurrent acute on chronic pancreatitis. ${ }^{3}$ When coupled with PD strictures, stone size, arrangement, and typical characteristics of being hard and spiculated with sharp edges often leads to PD occlusion and impaction. PD stone fragmentation and removal can reduce PD obstruction, thereby relieving upstream ductal pressure and alleviating pain. In cases where extracorporeal shock wave lithotripsy (ESWL) is not available, or in cases where obstructive stones cannot be fragmented after multiple ESWL sessions, EUS-PDD may be a viable option to drain the PD and relieve ductal hypertension-associated abdominal pain.

\section{Disconnected pancreatic duct}

Disconnected PD syndrome is a recognized complication of acute necrotizing pancreatitis, chronic pancreatitis, and abdominal trauma that results in a rupture of the main PD and viable pancreas secretions failing to drain into the gastrointestinal tract. Extravasated pancreatic secretions from a disconnected duct can result in complications including high-amylase ascites and pleural effusions, internal or external fistulas, and pseudocysts. Transpapillary stenting in conjunction with continued medical therapy has been reported to be successful in $55 \%$ to $84 \%{ }^{4-6}$ of patients, whereas surgical therapy, often a distal pancreatectomy, carries a relatively high morbidity and mortality. Percutaneous rendezvous therapies for PD duct disruption have been described, but are not routinely used. ${ }^{7}$ EUSPDD has been reported to be successful in cases of chronic pancreatitis with complete rupture of the main $\mathrm{PD}^{2}$ and in cases of PD leaks.

\section{Inability to access/cannulate the papilla by ERP}

Cannulation of the $\mathrm{PD}$ via the major papilla has a high success rate, recently reported to be between $90 \%$ to $98 \%{ }^{8}$ Failure of selective PD cannulation can occur due to an inability to identify the major papilla, stenosis after endoscopic biliary sphincterotomy or surgical sphincteroplasty, ampullary adenoma or tumor obscuring papillary visibility, history of ampullectomy, distorted gastric or duodenal anatomy, and/ or peridiverticular papilla location. The first EUS-guided rendezvous technique for $\mathrm{PD}$ access was reported in a patient with a peridiverticular, previous biliary sphincterotomy and a suprapapillary stricture that precluded ERP despite multiple attempts in 2002 . $^{9}$

\section{Postsurgical anatomy}

\section{Strictures postpancreatoduodenectomy}

Patients with a stenosis of the pancreatoenteric anastomosis may present with acute pancreatitis or epigastric abdominal pain and should be considered for endoscopic interventions for PD decompression. In general, pancreatoenteric anastomotic site strictures are more common after pancreatogastrostomy than pancreatojejunostomy, ${ }^{10}$ with pancreatojejunostomy strictures reported in approximately $5 \%$ to $11 \%$ of post Whipple patients, ${ }^{11,12}$ and up to $30 \%$ for pancreatogastrostomy. ${ }^{13}$ Pancreatic fistula accompanying pancreatoenteric stenosis or occlusion is also observed in $10 \%$ to $20 \%$ of these patients. ${ }^{14}$ ERP in postsurgical anatomy can be technically challenging because of the difficulty in reaching the pancreatoenteric anastomosis, identifying the anastomosis, and cannulating the PD even when it can be successfully located. In the largest published case series of 14 patients with attempted EUS guided PDD in patients who underwent postpancreatoduodenectomy, the rendezvous technique was successful in 11 of 17 procedures $(64.7 \%)$ performed. ${ }^{15}$

\section{ACCESSORIES}

\section{Needle: 19 gauge}

While accessing the PD can be achieved with both, small caliber 25- and 22-, and larger caliber 19-gauge needles, the indication for PD access, the degree of PD dilation and the presence of pancreatic parenchymal changes should all be taken into account. ${ }^{16,17}$ For example, in dilated PDs ( $\left.>5 \mathrm{~mm}\right)$ or for the placement of transluminal stents, the use of a 0.035 inch guidewire, which requires a 19-gauge needle, is most appropriate and frequently the instrument of choice. However, the larger 19-gauge needle may change the angle of the linear echoendoscope probe and might be more difficult to 
pass through a fibrotic pancreas. For pancreatography, smaller caliber needles can provide similar access with less risk of complications including leakage, bleeding or postprocedure pancreatitis. $^{18}$

In the largest case series of EUS guided PD stent placement for drainage reported by Fujii et al. ${ }^{19}$ (43 patients, 32 with technical success), a 19-gauge fine needle aspiration (FNA) needle (Cook Medical, Bloomington, IN, USA) was used for duct access. The second largest case series by Tessier et al. ${ }^{2}$ (36 patients, 33 with technical success) included either a 19or 22-gauge FNA needle (EUS-19-T or ECHO-1-22; Cook Medical), or inner needle of a cystoenterostome (Cystostome CST10; Cook Medical).

\section{Guidewires: 0.035 inch}

Similar to needle selection, the choice of guidewire should be tailored by their inherent properties (including stiffness, fluoroscopic and endoscopic visibility, and ease of over-thewire instrument exchange) and by the goals of the procedure. Transanastomotic or transampullary PD stenting and rendezvous procedures often require a stiffer, hydrophilic wire/ tip such as a 0.025 or 0.035 inch angled, or straight Visiglide (Olympus America, Center Valley, PA, USA) to bypass the anastomosis or ampulla with minimal kinking. The main benefit of utilizing smaller caliber 0.021 or 0.018 inch wires with either a 19- or 22-gauge needle is their flexibility, which can aid in maneuvering the wire though a tortuous duct and/ or bypassing ductal strictures. Unfortunately, these small wires often have reduced radiographic opacity, and the lack of stiffness makes over-the-wire instrument exchange and maintenance of the wire position more challenging. At the cost of reduced flexibility and increased PD insertion difficulty, stiff larger wires provide access that is associated with less kinking, increased fluoroscopic visibility, and increased stability for ease of subsequent instrument passage. This has led some experts to recommend transitioning from a 0.018 or 0.020 inch guidewire to a larger, stiffer wire ( $0.035 \mathrm{inch}$ ) in preparation for stenting or rendezvous. ${ }^{18}$ Along these lines, in the Tessier et $\mathrm{al}^{2}$ case series, when a smaller wire $(0.020 \mathrm{inch})$ was used, it was subsequently exchanged for a larger 0.035 inch wire. In the Fuji et al. ${ }^{19}$ case series, either a 0.035 or 0.025 inch, both straight and angled hydrophilic versions (Glidewire, Jagwire; Boston Scientific, Natick, MA, USA) were used.

\section{Dilation devices}

EUS guided antegrade PD stenting requires the bowel luminal (gastric, duodenal, or jejunal)-PD fistula tract to be dilated after needle puncture and wire insertion. A wide array of endoscopic tools including hydrostatic balloons (Titan biliary dilation Balloon, Cook Medical; or $4 \mathrm{~mm}$, Hurricane Biliary
Dilation Balloon, Boston Scientific), tapered catheters (5- to 7-Fr, Sohendra; Cook Medical), cannulas (Proforma; Conmed Endoscopic Technologies, Utica, NY, USA), and/or diathermic catheters such as a needle knives can be used to dilate the tract. ${ }^{19}$ To date, there have been no comparative trials to evaluate the success and complication rates of tract dilation methods. The use of diathermic catheters remains controversial. Multiple experts recommend their use as a last resort because of concerns of cautery-related adverse events, ${ }^{18,20}$ while others have reported personal experience with less peripancreatic juice leak relative to balloons. ${ }^{21}$ Endoscopists, therefore, tend to rely upon personal experience when deciding what equipment to use and often, multiple devices are serially employed.

\section{Plastic stents}

PD stents that have been used include straight as well as single and double pigtail stents. In the largest case series from Japan, 7 and 5 Fr plastic stents were most commonly used with single cases of an $8 \mathrm{~mm}$ self-expandable metal stent and $5 \mathrm{Fr}$ naso-PDD (with subsequent exchange to a 7 Fr plastic stent). ${ }^{15}$ Stent dysfunction can occur either through migration or occlusion, and although not statistically significant, stent migration occurred more frequently with straight plastic stents than with double pigtail plastic stents in one of the largest studies on EUS-PDD. ${ }^{20}$ Recently, a new, single pigtail 7-Fr plastic stent was developed specifically for EUS-guided PD placement by Itoi et al. ${ }^{22}$ The stent has a tapered tip, four internal flanges (two in the distal end and two at the proximal end), and a single external pigtail (total length of $20 \mathrm{~cm}$ and effective length of $15 \mathrm{~cm}$ ). The initial feasibility report of eight patients achieved technical and treatment success in $100 \%$ of cases.

\section{Metal stents}

Covered metal stents were initially not recommended for use due to concerns of PD obstruction; however, Oh et al. ${ }^{23}$ recently described EUS-PDD using a novel fully covered self-expandable metal stent (FCSEMS) with anti-migration properties in 25 patients with "painful obstructive pancreatitis" after failed ERP. This modified FCSEMS (commercially available, silicone coated, nitinol wire, 6 or $8 \mathrm{~mm}$ diameter, 6 to $10 \mathrm{~cm}$ in length; M.I. Tech, Seoul, Korea) with proximal and distal anchoring flaps was placed into either a pancreatogastric or pancreatoenteric fistula. EUS-guided pancreatogastrostomy $(n=23)$, pancreatoduodenostomy $(n=1)$, and pancreatojejunostomy $(n=1)$ were performed. EUS-PDD had 100\% technical and clinical success rates. Pain scores improved significantly after FCSEMS placement ( $p=0.001$ ). Early mild grade adverse events occurred in five patients (20\%), four with self-limited abdominal pain and one with minor bleeding. 


\section{TECHNIQUES FOR EUS-PDD}

Pre-procedural considerations that should be made include giving the patient intravenous antibiotics, placing the patient in the prone position to allow for easier recognition of the PD direction under fluoroscopy, considering general anesthesia for lengthy procedures, utilizing $\mathrm{CO}_{2}$ insufflation, and using a therapeutic channel curvilinear echoendoscope to allow for the passage of large caliber stents.

Although a dilated PD is not a requirement for this procedure, it does appear to carry an advantage. The optimal access site is selected by choosing the location with the shortest distance between the bowel lumen and the PD, an absence of interposed vasculature, and maximal stability with an angle to allow tract dilation and device deployment. In the majority of cases published to date, the optimal PD access was obtained with a transgastric approach. After the PD is identified, it is punctured using an FNA needle. Once the needle has entered the $\mathrm{PD}$, contrast is injected and fluoroscopy is used to obtain a pancreaticogram and ensure PD access. Next, a guidewire is passed through the FNA needle into the PD, and then, depending on the technique, it is advanced and looped into the duodenum or further into the PD with care taken to prevent wire dislodgement. Once the $\mathrm{PD}$ puncture and guidewire has been performed, fluoroscopic imaging is used to prevent the loss of guidewire access and ensure appropriate scope position.

\section{Pancreatic drainage techniques}

\section{Rendezvous (retrograde)}

A rendezvous, also known as retrograde, stent placement technique uses a curvilinear array echoendoscope to puncture the PD to gain access. After obtaining access, the echoendoscope is removed and exchanged for a side-viewing duodenoscope (in patients with unaltered anatomy) or a forward-viewing endoscope (in patients with surgically altered anatomy, e.g., Roux-en-Y resection). After the endoscopes are exchanged, a stent can be placed retrograde from the gut lumen via the papilla or an anastomosis (transpapillary or transanastomotic) into the PD.

The guidewire is inserted into the main PD and passed in an antegrade fashion across the major papilla or stenotic pancreatoenteric anastomosis. Looping of the guidewire in the small bowel is necessary to avoid guidewire migration and loss of access. The echoendoscope is removed and a duodenoscope or forward viewing scope (colonoscope or balloon assisted enteroscope) is advanced to the major papilla or stenotic pancreatoenteric anastomosis and the intraluminal guidewire is captured with a snare, grasper, or biopsy forceps. The wire is then pulled through the endoscope until both ends of the guidewire are accessible to the endoscopist (one end via the endoscope therapeutic channel, the other end is free). Retrograde PD stenting can be completed subsequently over the endoscope therapeutic channel wire as routinely performed. If stenting of the distal PD is necessary, a second guidewire may be inserted in a retrograde fashion beyond the PD access site with a double lumen catheter over the first wire. There are reports of increased technical success when two endoscopists perform the rendezvous method together; however, this approach is not routinely practiced. ${ }^{24}$

\section{Direct drainage (antegrade)}

In the antegrade or direct drainage technique, PD access and stent placement is completed with the curvilinear array echoendoscope alone (Fig. 1). In this technique, a stent is deployed from the bowel into the PD either terminating across the obstruction/papilla/anastomosis (transanastomotic or transpapillary) or not (transluminal). For the antegrade technique, tract dilatation is obligatory to pass the stent through the gastrointestinal tract wall into the PD. In cases where the guidewire does not initially bypass the obstruction or papil$\mathrm{la} /$ anastomosis into the small bowel, the use of the dilation catheter via the transluminal tract may be attempted to aid the passage of the guidewire. Ideally, stent placement would be transanastomotic or transpapillary, with the distal portion of the stent inside the small bowel and the proximal portion lying in the stomach. However, bypassing the obstruction/ papilla/anastomosis with the guidewire may not always be successful, in which case, the distal portion of the stent can be left inside the PD with the proximal portion in the stomach (transluminal). Relative to transpapillary or transanastomotic stents, transluminal stents are at a higher risk of stent migration and resultant leakage of pancreatic fluids. Consequently, transluminal placement is reserved only for when transpapillary or transanastomotic stent placement is not possible.

\section{Tips and tricks}

\section{Tract dilation}

Tract dilation is considered to be one of the most challenging aspects in EUS-guided PD stenting. This can be due to misaligned pushing force/needle axis angle, or gastric wall thickness, and/or the fibrotic nature of the pancreatic parenchyma in chronic pancreatitis. ${ }^{15}$ We try to avoid the use of diathermic catheters (e.g., needle knife) to minimize cautery-associated complications. Dilatation with the sheath of the EUS-FNA needle may be used as an initial dilation device. In addition, avoiding a perpendicular angle of approach, minimizing gastric lumen-PD distance, and minimizing tract dilation diameter (5 to $7 \mathrm{Fr}$ catheters or $4 \mathrm{~mm}$ balloons) may 

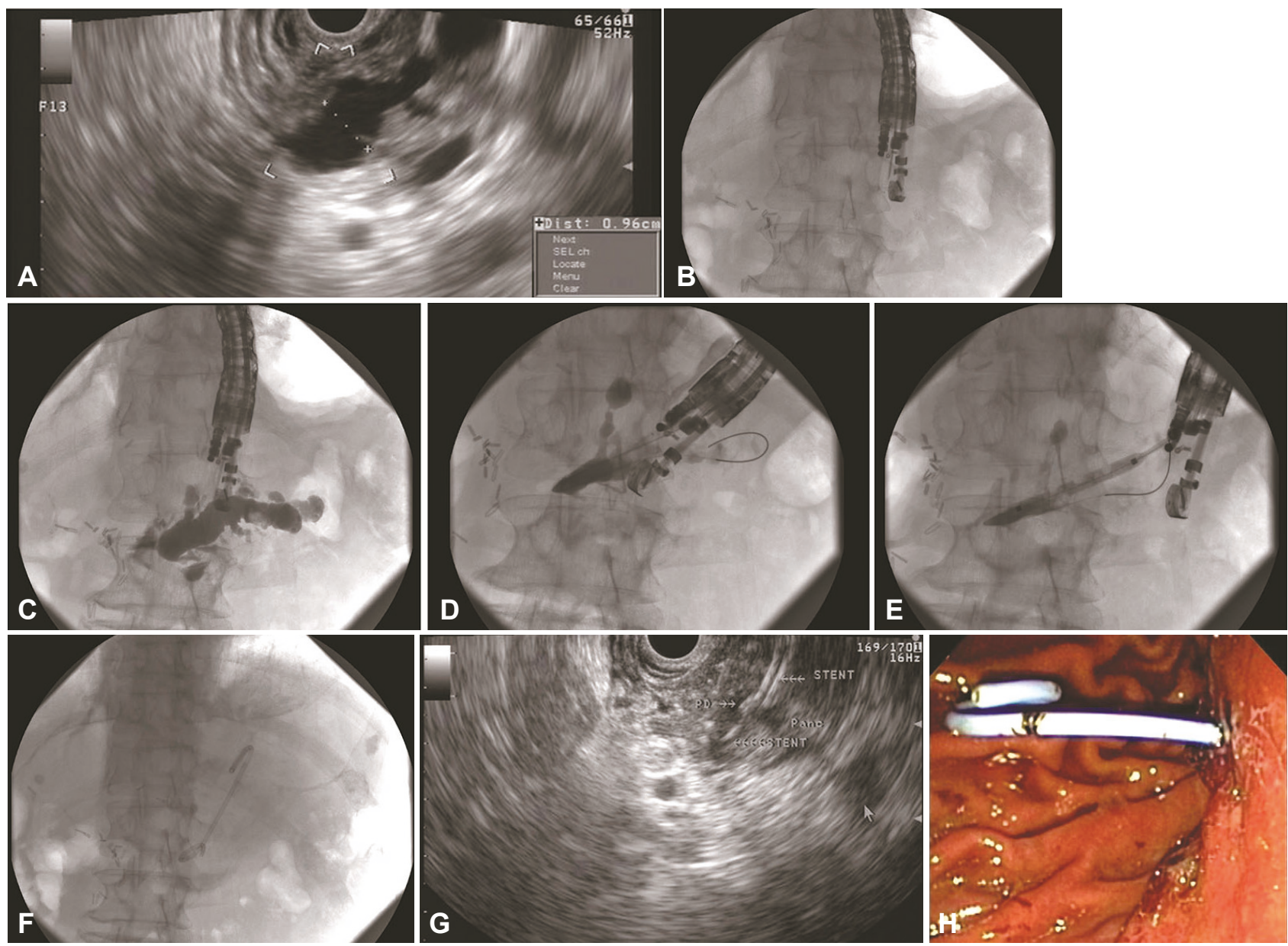

Fig. 1. Endoscopic ultrasound (EUS)-guided anterograde pancreatic duct drainage and stenting in a patient with Whipple surgery and anastomotic stenosis. (A) EUS imaging revealing a dilated main pancreatic duct to $10 \mathrm{~mm}$ in maximum diameter. (B) The curvilinear echoendoscope is positioned to puncture the main pancreatic duct. (C) A 19 gauge EUS-fine needle aspiration is advanced into the main pancreatic duct and a pancreatogram is obtained revealing a dilated pancreatic duct. (D) A 0.035-inch guidewire is advanced into the main pancreatic duct; however, it is unable to bypass the anastomosis and is coiled in the distal pancreatic duct. (E) The pancreatogastrostomy tract is dilated with a $4 \mathrm{~mm} \times 4 \mathrm{~cm}$ dilation balloon. (F) A $7 \mathrm{Fr}$ by $4 \mathrm{~cm}$ double pigtail plastic stent was then placed over the wire across the pancreatogastrostomy. The stent position is confirmed endosonographically $(G)$ and endoscopically $(H)$.

decrease the risk of pancreatic juice leakage.

\section{Avoiding side branch ducts}

A frequent indication for EUS-guided PD drainage is chronic pancreatitis; thus, there are often multiple dilated side branches making passage of the guidewire to the papilla difficult. This can often be remedied by either switching to a different guidewire (alternative type, size, or angled/straight) or changing the angle of the needle's approach to the PD.

\section{Crossing obstructions and strictures}

Advancing the guidewire across the obstruction/papilla/ anastomosis can be challenging. Tricks to employ include removing and advancing the guidewire again, changing the needle angle and/or the guidewire, and perhaps magnifying or rotating fluoroscopy to gain additional views. As in ERP, one can also inflate balloons near the obstruction to help di- rect guidewire passage through the obstruction.

\section{Avoiding wire stripping}

When the PD and echoendoscope needle are at a tight angle, there is an increased risk of shaving off the wire tip when withdrawing the guidewire into the needle. In order to minimize this risk, care should be taken to straighten out any acute angles prior to wire withdrawal and never pulling against resistance. If the wire cannot be withdrawn easily, then we suggest removing both, the guidewire and needle, together.

\section{OUTCOMES}

\section{Technical success}

In 2015, Fujii-Lau and Levy ${ }^{20}$ summarized the current literature on EUS-guided PD drainage, reviewing the pub- 
lished experience of 222 patients. Including both antegrade and rendezvous techniques, technical success was achieved in $170 / 222$ patients $(76.6 \%)$. A similar review by Itoi et al. ${ }^{18}$ in 2013 reported a technical success rate of $>70 \%$ in 75 patients using the antegrade technique and a range of success rates from $25 \%$ to $100 \%$ in 52 patients with the rendezvous technique. The largest experience in the rendezvous cohort was 20 patients with a lower reported technical success rate of $48 \%$.

In the largest series of attempted EUS guided PD stent placement, which involved 43 patients, technical success occurred in 32 patients $(74 \%)$, with 18 undergoing antegrade EUS-PDD (13 transpapillary or transanastomotic vs. five transluminal) and 14 undergoing retrograde/rendezvous EUSPDD. ${ }^{19}$ ERP performed during the same procedure was the only statistically significant factor associated with failure of stent placement. Long-term outcomes (defined as a minimum 1 year of follow-up) were available for 29 patients. Complete clinical success defined as all symptom resolution occurred in $69.6 \%$ and partial symptom resolution (improved severity or frequency of symptoms) was found in the remaining $30.4 \%$. In a 12 patient case series, $29 \%$ of patients underwent surgery within a follow up range of 4 weeks to 3 years. ${ }^{25}$

\section{Adverse events}

In the recently published summary of available EUS-PDD case series, complications were documented in 42 of 222 patients $(18.9 \%){ }^{20}$ The most frequent complication was abdominal pain occurring in 17 patients (7.7\%) followed by pancreatitis in seven patients (3.2\%). Additional less frequently reported complications included four patients with bleeding, two patients each with perforation, peripancreatic abscess, or shearing of the guidewire. A single patient was reported to develop fever, pneumoperitoneum, pseudocyst, aneurysm, or perigastric fluid collection. In the largest published, single center experience, the adverse event rate was lower at $6 \%$, with one episode of pancreatitis requiring an 11 day admission, one abscess requiring EUS drainage, one retained sheared wire, and 13 patients with abdominal pain requiring admission (median length of stay, 2 days). ${ }^{19}$

Stent dysfunction, defined as migration or occlusion, has been reported to occur in $25 \%{ }^{19}$ to $55 \%$ of cases. ${ }^{2,26}$ In the case series by Fujii et al., ${ }^{19}$ stent dysfunction occurred in eight patients (25\%) including five cases of stent migration, of which four of five occurred with straight plastic stents. Although not statistically significant, migration occurred in $23 \%$ of straight stents compared to $9 \%$ of double pigtail stents, leading the endoscopists to favor the use of double pigtail stents. ${ }^{19}$

\section{CONCLUSIONS}

As EUS continues to offer an increasingly diverse repertoire of therapeutic interventions, the possibility to drain PDs when ERP is not successful, and is emerging as a minimally invasive, non-surgical technique. Although data has demonstrated the procedure can be safe and effective, EUS-guided PD drainage remains one of the most technically challenging therapeutic EUS interventions, as evidenced by the multiple considerations on device selection and risk of severe complications. At this time, we advocate that this procedure should only be performed in appropriately selected patients by experienced endoscopists trained in both, EUS and ERP, with welltrained surgical back-up available.

\section{Conflicts of Interest}

I.W. has been a consultant for Cook Medical and Olympus America.

\section{REFERENCES}

1. Dumonceau JM, Delhaye M, Tringali A, et al. Endoscopic treatment of chronic pancreatitis: European Society of Gastrointestinal Endoscopy (ESGE) Clinical Guideline. Endoscopy 2012;44:784-800.

2. Tessier G, Bories E, Arvanitakis M, et al. EUS-guided pancreatogastrostomy and pancreatobulbostomy for the treatment of pain in patients with pancreatic ductal dilatation inaccessible for transpapillary endoscopic therapy. Gastrointest Endosc 2007;65:233-241.

3. Ammann RW, Muench R, Otto R, Buehler H, Freiburghaus AU, Siegenthaler W. Evolution and regression of pancreatic calcification in chronic pancreatitis. A prospective long-term study of 107 patients. Gastroenterology 1988;95:1018-1028.

4. Bakker OJ, van Baal MC, van Santvoort HC, et al. Endoscopic transpapillary stenting or conservative treatment for pancreatic fistulas in necrotizing pancreatitis: multicenter series and literature review. Ann Surg 2011;253:961-967.

5. Brennan PM, Stefaniak T, Palmer KR, Parks RW. Endoscopic transpapillary stenting of pancreatic duct disruption. Dig Surg 2006;23:250-254.

6. Varadarajulu S, Noone TC, Tutuian R, Hawes RH, Cotton PB. Predictors of outcome in pancreatic duct disruption managed by endoscopic transpapillary stent placement. Gastrointest Endosc 2005;61:568-575.

7. Irani S, Gluck M, Ross A, et al. Resolving external pancreatic fistulas in patients with disconnected pancreatic duct syndrome: using rendezvous techniques to avoid surgery (with video). Gastrointest Endosc 2012;76:586-593.

8. Benage D, McHenry R, Hawes RH, O’Connor KW, Lehman GA. Minor papilla cannulation and dorsal ductography in pancreas divisum. Gastrointest Endosc 1990;36:553-557.

9. Bataille L, Deprez P. A new application for therapeutic EUS: main pancreatic duct drainage with a "pancreatic rendezvous technique". Gastrointest Endosc 2002;55:740-743.

10. Amano H, Takada T, Ammori BJ, et al. Pancreatic duct patency after pancreaticogastrostomy: long-term follow-up study. Hepatogastroenterology 1998;45:2382-2387.

11. Morgan KA, Fontenot BB, Harvey NR, Adams DB. Revision of anastomotic stenosis after pancreatic head resection for chronic pancreatitis: is it futile? HPB (Oxford) 2010;12:211-216.

12. Reid-Lombardo KM, Ramos-De la Medina A, Thomsen K, Harmsen WS, Farnell MB. Long-term anastomotic complications after pancreaticoduodenectomy for benign diseases. J Gastrointest Surg 2007;11:1704- 
1711.

13. Takano $\mathrm{S}$, Ito $\mathrm{Y}$, Oishi $\mathrm{H}$, et al. A retrospective analysis of 88 patients with pancreaticogastrostomy after pancreaticoduodenectomy. Hepatogastroenterology 2000;47:1454-1457.

14. Kikuyama M, Itoi T, Ota Y, et al. Therapeutic endoscopy for stenotic pancreatodigestive tract anastomosis after pancreatoduodenectomy (with videos). Gastrointest Endosc 2011;73:376-382.

15. Kurihara T, Itoi T, Sofuni A, Itokawa F, Moriyasu F. Endoscopic ultrasonography-guided pancreatic duct drainage after failed endoscopic retrograde cholangiopancreatography in patients with malignant and benign pancreatic duct obstructions. Dig Endosc 2013;25 Suppl 2:109-116.

16. Kahaleh M, Hernandez AJ, Tokar J, Adams RB, Shami VM, Yeaton P. Interventional EUS-guided cholangiography: evaluation of a technique in evolution. Gastrointest Endosc 2006;64:52-59.

17. Mallery S, Matlock J, Freeman ML. EUS-guided rendezvous drainage of obstructed biliary and pancreatic ducts: report of 6 cases. Gastrointest Endosc 2004;59:100-107.

18. Itoi T, Kasuya K, Sofuni A, et al. Endoscopic ultrasonography-guided pancreatic duct access: techniques and literature review of pancreatography, transmural drainage and rendezvous techniques. Dig Endosc 2013;25:241-252.

19. Fujii LL, Topazian MD, Abu Dayyeh BK, et al. EUS-guided pancreatic duct intervention: outcomes of a single tertiary-care referral center ex- perience. Gastrointest Endosc 2013;78:854-864.

20. Fujii-Lau LL, Levy MJ. Endoscopic ultrasound-guided pancreatic duct drainage. J Hepatobiliary Pancreat Sci 2015;22:51-57.

21. Giovannini M. EUS-guided pancreatic duct drainage: ready for prime time? Gastrointest Endosc 2013;78:865-867.

22. Itoi T, Sofuni A, Tsuchiya T, et al. Initial evaluation of a new plastic pancreatic duct stent for endoscopic ultrasonography-guided placement. Endoscopy 2015;47:462-465.

23. Oh D, Park do H, Cho MK, et al. Feasibility and safety of a fully covered self-expandable metal stent with antimigration properties for EUS-guided pancreatic duct drainage: early and midterm outcomes (with video). Gastrointest Endosc 2016;83:366-373.

24. Vila JJ, Pérez-Miranda M, Vazquez-Sequeiros E, et al. Initial experience with EUS-guided cholangiopancreatography for biliary and pancreatic duct drainage: a Spanish national survey. Gastrointest Endosc 2012;76:1133-1141.

25. Will U, Fueldner F, Thieme AK, et al. Transgastric pancreatography and EUS-guided drainage of the pancreatic duct. J Hepatobiliary Pancreat Surg 2007;14:377-382.

26. Ergun M, Aouattah T, Gillain C, Gigot JF, Hubert C, Deprez PH. Endoscopic ultrasound-guided transluminal drainage of pancreatic duct obstruction: long-term outcome. Endoscopy 2011;43:518-525. 